\title{
An Improved MAC Address Based Intrusion Detection and Prevention System in MANET Sybil Attacks
}

\author{
C.A. Gokula Krishnan and Dr.A. Suphalakshmi
}

\begin{abstract}
A Mobile Adhoc Network is a communication medium that does not rely on secure infrastructure. It is a group of independent mobile nodes that can transfer to communicate each other via radio waves. These networks are fully circulated, and can work at any apartment without the help of any permanent infrastructure as access points or base locations. As in ad- hoc network communication intermediate in network medium is air so it would be easy for invader to fetch information from air intermediate using sniffing simulation software tool. There is an occurrence which causes so abundant obliteration to a network called Sybil attack as intrusion. In the Sybil attack a each node presents numerous fake characteristics to other swellings in the network. In this paper, we realized to in tentds the Sybil Attack Detection Based on MAC address classification technique which is used to detect the Sybil nodes in the network and also prevent it. The in defender uses dissimilar identities at the same time to create attacks. A solitary attacker could imaginary nodes to report the being of a false bottleneck in circulation. MANETs are mostly related to illegally assembly sensitive evidence info about mobile nodes. To transmit between a source and its destination to analyses the intrusions, to treasure the consequence on data and broadcast time on network communication.
\end{abstract}

Keywords--- Intrusion Detection, Network Security, Network Communication, MANET.

\section{INTRODUCTION}

A D HOC network communication is emergence expertise of wireless communication for mobile transmission medium nodes. In an ad hoc network communication, nearby malicious network is no secure substructure such as base positions or mobile swapping centers. Mobile swellings that are inside each other's radio range communicate straight via wireless links, while individuals that are far distinctly rely on other nodules to relay communications as routers. Node elasticity and flexibility in an ad hoc network reasons frequent changes of the system in network topology.

Due to organization less nature of MANET and as nearby is no central expert to preserve and regulator the system makes it vulnerable to various attacks. Ad hoc systems can be used for battleground emergency, law implementation, and

C.A. Gokula Krishnan, ME (CSE), Paavai Engineering College, Pachal, Namakkal, Tamil Nadu. E-mail:gokulkannanking@gmail.com

Dr.A. Suphalakshmi, M.E., Ph.D., Assistant Professor (CSE), Paavai Engineering College, Pachal, Namakkal, Tamil Nadu.

DOI: 10.9756/BIJRCE.8315 liberation missions. Nodes in MANET communicate with both other on the basis of exclusive identity that arrangements the one to one plotting between an distinctiveness and an entity and that is Frequently assumed either obliquely or explicitly by numerous protocol mechanisms to be implemented, hence two individualities implies two separate nodes. But the malicious nodes can illicitly to act intrusion be claim multiple individualities and violate this one-to-one charting of identity and entity attitude. Sybil attack is an occurrence which uses several personalities at a period and increases lot of miscalculations among the nodes of a net or it may use individuality of other genuine nodes present in the network and makes false expression of that bulge in the network. Like this, it interrupts the communication between the nodes of the communication network.

To consume protected communication it is essential to eliminate the Sybil nodes from the net. The subsequent goals must be satisfied by security algorithm used to notice the attack:

1) Authentication: The resources that each and every node, contributing in communication must be honest and authentic node.

2) Availability: All facilities should be obtainable all the time to altogether the nodes for the appropriate functioning and security of the network.

3) Integrity: It gives the guarantee that the data conventional by the receiver will be same as the data send by the despatcher.

4) Confidentiality: It proceeds that convinced data is only neighboring by the official users.

5) Non-repudiation: It means data sender and receiver cannot deny the communication that they didn't send or receive the data

A intrusion combined with exampled malicious node $\mathrm{X}$ along with its three Sybil nodes (A, B and C). If this malicious node transmissions with any authentic node by donating all its identities, the genuine node will have impression that it has connected with four dissimilar nodes. But in actual, there happens only one bodily node with numerous IDs.

Commonly, network-based intrusion identification systems can be categorized into two main categories, specifically misuse based discovery the systems and anomaly-based revealing systems. Misuse-based detection schemes detect attacks by observing network activities and observing for competitions with the prevailing attack autographs. In spite of consuming high exposure rates to known attacks and low false encouraging rates, misuse-based detection systems are easily 
evaded by any new attacks and even variants of the existing attacks. Furthermore, it is a complicated and labor intensive task to keep signature database updated because signature generation is a manual process and heavily contains network security expertise. Investigation community, consequently, started to explore away to achieve novelty-tolerant detection arrangements and developed a more progressive concept, namely abnomaly based detection. Outstanding to the principle of attack detection, which observers and flags any network happenings presenting substantial deviation from legitimate traffic profiles as suspicious objects, anomaly-based detection techniques show more auspicious in detecting zeroday interruptions that exploit previous unidentified system vulnerabilities

Moreover, it is not inhibited by the expertise in system security, due to the circumstance that the profiles of genuine behaviors are developed founded on techniques, such as statistics mining machine learning and arithmetical reviews statics analysis. However, these projected systems usually suffer from high false optimistic rates because the relationships between features/attributes are essentially neglected or the methods do not achieve to fully adventure these correlations. Current studies have focused on feature connection analysis.

\section{RELATED WORK}

A Novel Mechanism for Detection of Sybil Attack in MANETs [1], Mobile adhoc networks provide communication between wireless nodes in the absence of fixed infrastructure[1]. MANETs unlike wired networks because of lack of central authority and mobile nature are more vulnerable to attacks. Sybil attack is the type of attack in mobile adhoc networks in which attacker intrudes in the network and acts with multiple identities to disrupt the normal and trustworthy communication between nodes[2]. In this Novel mechanism is proposed that ensures the detection of both Simultaneous Sybil attack and Join and Leave Sybil attack in the network.

Secure Verification Protocol to Detect Sybil Attacks in MANETs[2]recommend an end-to-end secure communiqué arrangement communication structure for W2T in WSNs in which we shadow an unequal approach for secure verification and key organization using to prevent IDS. In this scheme, a boundless communicating part of the work for packet identification and access regulator is removed to a entry between a WSN and the Internet to decrease the load and energy ingesting in the device nodes[3]avoiding Sybil attack and malicious rapidly and in suitable to irresponsible nodes (malicious node). They presentation a novel and secure verification of nodes as rapidly as they originates in to the network (checks the identity of a new node) and then inspection the RSS value of node uninterruptedly and accurately perceiving the sybil identity in the network.

Mobile Based Sybil Attack in IDS detection using mobile ID on the Mobile ADHOC Network. Mobility is often a problem for providing security services in ad hoc networks. Mobility can be used to increase security [4]y. A benefit of such a network is that no fixed infrastructure is compulsory. In the Sybil attack a solitary node contributions multiple fake individualities to other nodes in the network. Sybil attacks pose a countless hazard problems to distributed systems like peer-to-peer systems and topographical routing protocols. In the technique, use unreceptive ad hoc individuality method and key delivery. The sybil attack in sensor networks: analysis \&defenses[5] Detection can be complete by a single node, or that numerous confidential nodes can join to recover the accuracy of exposure. In Sybil attacks position a great danger to decentralized schemes like peer-to-peer systems communication and geographic routing protocols.

Proposed Lightweight Sybil Attack Detection Technique in MANET [6].A established of mobile nodes which can intersect straight with additional nodes inside its broadcast range and use multihop directing for nodes external its transmission range is called Mobile Ad hoc Network (MANET). The substructure less nature (bandwidth, memory and battery power) of MANET makes it vulnerable to numerous attacks. Due to the multifaceted nature of MANETs and its reserve constraint nodes, there has always been a need to develop lightweight security solutions. The MANETs communication that require a exclusive discrete and strongminded independence per node in teaching for their safety measures to be workable with affordable links in nodes, Projected the communication Insubstantial Sybil Attack Detection Technique in MANET be described as IDS.[7]Sybil attacks generate a serious danger to such networks.

A Sybil attacker can whichever create more than one individuality on a single physical scheme in order to launch a harmonized attack on the network or can adjustment identities in order to deteriorate the discovery process, thereby indorsing lack of responsibility in the net. It is strongly wanted to detect Sybil attacks and eliminate them from the network. This determined a lightweight scheme to notice the new individualities of Sybil attackers deprived of using central trusted third party or any supplementary hardware, such as indicator antennae or a topographical identification system.

Mobile Ad-Hoc network (MANET) is a temporary infrastructure less network [9]. This network is shaped by merging some customary of wireless mobile hosts. The swarm is called as a node which enthusiastically founds their own network. In MANET all the nodes activates in accommodating fashion. Due to their convinced inherently susceptible characteristics, there are numerous potentials of the attacks in MANET. Every time interruption prevention portion not certain to identified work. Mobile-id Based Sybil Attack discovery on the Mobile ADHOC Network[11] screen is successful on in the system and appearance for intrusion using Intrusion Detection System (IDS).

In this IDS construction multilayer requirement based discovery engine is used. This screens the transport, system and data link level of the protocol stack. It arbitrarily traverses a system and discovery outs that on which node which attack is revealed the mitigation occurred. 


\section{Proposed Mac AdDRESS BASEd IDS IN SybiL ATTACKS}

In Proposed System is for detection the mitigation and prevention of Sybil attack, any node can surprise the discovery for Sybil node. In our circumstance despatcher node starts recognition for Sybil node previously it sends the packets which its covers to the receiver node. Firstly sender node transmission a appeal packet which in reappearance needs a reply communication which comprehend to find logical (IP) address and identified physical address (MAC).Despatcher nodes preserve a table for that and payments if a node with similar physical address answer with different rational address then the node with dissimilar logical identity is professed as a Sybil node and the despatcher node selects another path for distribution packets to destination.

A Mobile ad hoc network is composed of mobile, wireless devices, referred to as nodes that communicate only over a shared broadcast channel. An advantage of such a network is that no fixed infrastructure is required: a network for routing data can be formed from whatever nodes are available. Nodes forward messages for each other to provide connectivity to nodes outside direct broadcast range. Ad hoc routing protocols are used to find a path end-to-end through the cooperative network. In the Sybil attack the communication possess to represents a single node offerings manifold fake characteristics to other bumps that covers in the network. Sybil attacks pose a great threat to decentralized systems like peer-to-peer networks and geographic routing protocols. In our proposed method, we have using passive ad hoc identity method and key distribution. The efficient parameters are to be taken for differentiate the results on network. Improve the network overall performance and secure data transmission on the network.

\section{A. Contextual Security Threads}

The security threats can usually as content based with security and appropriate intent security. For the reviews of content security hazard, the adversary efforts to detect the satisfied of the packets sent in the network to study the detected data and the characteristics and positions of the source nodes. This safety threat can be contradicted by encoding the packets' insides and using aliases instead of the real identities. For the background security threat, the challenger eavesdrops on the system transmissions and uses traffic examination techniques to presume sensitive info, including whether, the mitigation occurs and where the data are composed. Actually, the performance of packet broadcast itself discloses material uniform if the packets are powerfully encrypted and the challenger could not understand them.

\section{B. Synchronization of Complexity in Overlay Position}

Manet networks most often have a much more complicated topology than the simple examples and not all Adhoc mitigation nodes that can communicate with medium of IDS nodes with each other directly. Thus, multi-hop management which the communication nodes which communication which its is required, which adds a supplementary coating of difficulty. Clearly, this might be evaded by using an intersection network which delivers virtual to synchronize, single-hop communication from every adhoc node to a single master node.

\section{System Architecture}

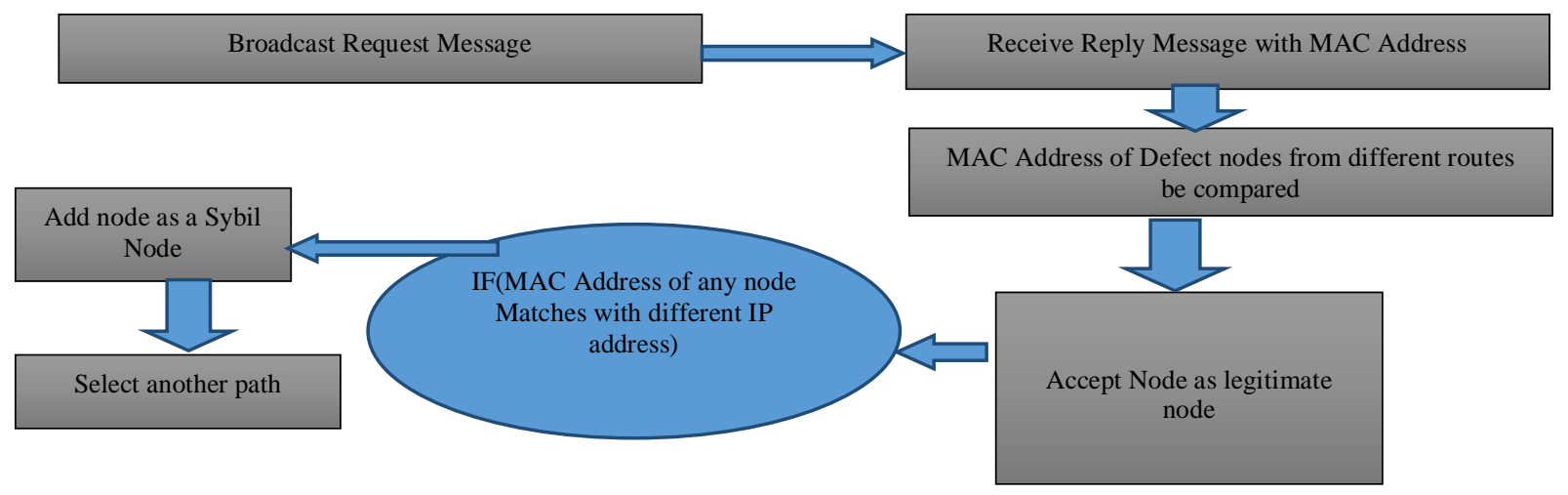

Figure 1: Architecture Diagram for Proposed System

\section{Algorithm}

Each node in the network is assigned with the universally unique identifier (UUID) during the registration process of the network. Admin stores each node UUID. Universally unique identifier UUIDs version 4 is used because of its randomness. The total size of ID is 128 bits; out of it 122 were random bits and remaining 4 for version and 2 for reserved bits. UUID.

Step1: Every node request for its neighboring nodes

Step 2: Then each node checks with its INFO_TABLE which contains neighboring nodes UUID. If neighboring nodes UUID mismatches, then it informs to the admin.

Step 3: If every UUID where unique, then a source node request for a destination node from the admin.

Step 4: After getting destination node source node will encryption the data and transmit to the destination. Finally destination node decrypts the data.

Use these algorithm at procedures to transfer the data in foundation to destination deprived of any injury or loss as well as every node to have the neighbor's node discourse. Be subject to intrusion on the address the data will be communicated in to correct endpoint. If they have any 
package loss are some crash on network immediately to notify the server to halt the data and upholding source node information and heading information of message. It payments the users using persons details whether they are assailants or normal user. Hacker's material has not been transported to destination. Destination has not been reception any attacker information. In our projected method to use protected and avoid the attacking system on the network to protect the communication under intruders.

\section{Timing Issue}

A network communication broadcast procedure is called static node IDS recover in the forward/non-forward status of each node is determined on the static view only; otherwise, it is dynamic. The static broadcast protocol is a special case of the dynamic one. The difference is that the forward node set derived from static views can be used in any broadcasting while the one derived from dynamic views is normally used in a specific broadcasting.

\section{Routing Prevention Schemas Representation}

Routing-based schemes try to preserve source nodes' location security by sending packets through different routes instead of one route, to make it infeasible for adversaries to trace back packets from the Sink to the source node because they cannot receive a unremitting movement of packets. However, if the challenger's overhearing variety is larger than the communicate nodes' broadcast range, the probability of capturing a big ratio of the packages sent from a source node significant that meaningfully intensifications of malicious. It is exposed that if the adversary's eavesdropping range is three epochs the relay nodes transmission range, the probability of locating pandas is as high as 0.97 . Moreover, if pandas stay for some time in one location, the adversary may capture enough number of packets to locate the pandas even if the packets are sent through different routes.

\section{E. Energy Consumption on Network}

The MAC Addressed protocol must preserve the resources of every node in the network. A single node failure in relay networks is usually unimportant if it does not lead to a loss of sensing and communication coverage; ad-hoc networks, instead, are oriented towards personal communication and the loss of connectivity to any node is significant. In the routing protocol design of mobile nodes, many issues need to be considered in order to offer many important properties such as scalability, QoS support, security, low power consumption and so on.

\section{RESUlT AND DisCUSSION}

In our proposed scheme for the computation of the MAC Based IDS method computes process in network parameters about the number of users registered to communicate in this group and simply selects a source and destination from set communication to detect the intrusions. The overhead produced by this calculation gives optimized result and complexity is less compare to other methods.

The proposed intrusion detection system for mobile adhoc network has been implemented and tested for its efficiency.
The approach has been implemented in

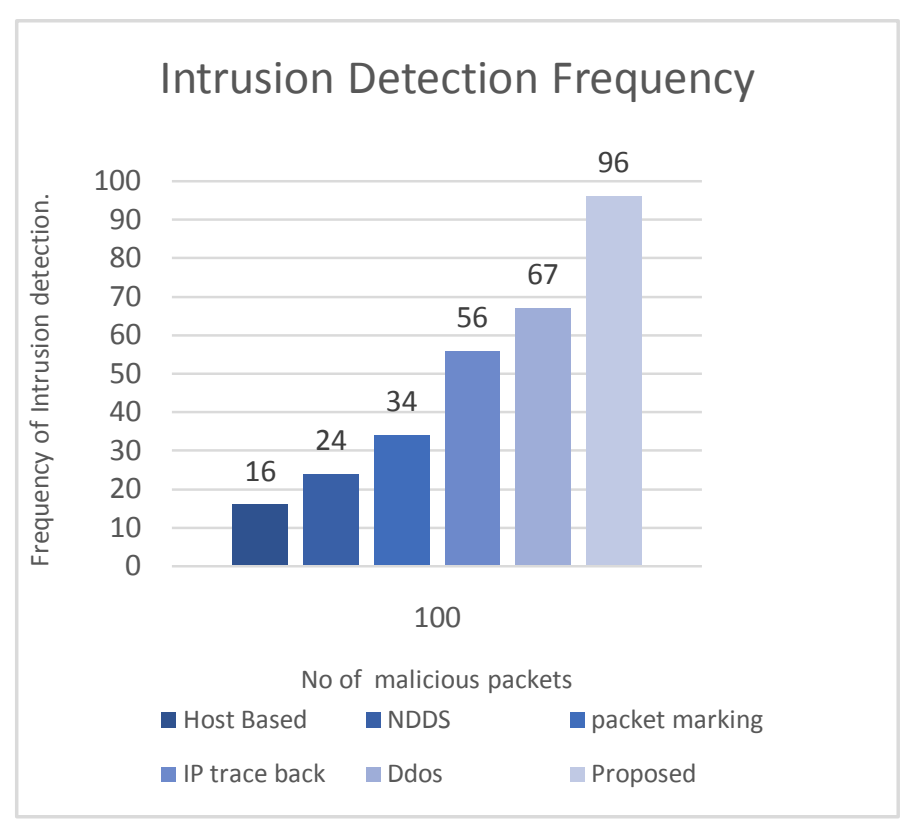

Graph 1: Shows the Frequency of Detection of Malicious Packet

The graph 1 shows the result of the proposed system in finding malicious packet and if there are 100 packets which are malicious arrived on time, then the graph shows the frequency of detection of malicious packet. It is very clear that the proposed system identifies the more malicious packet compare to other host based and activity pattern based intrusion detection systems.

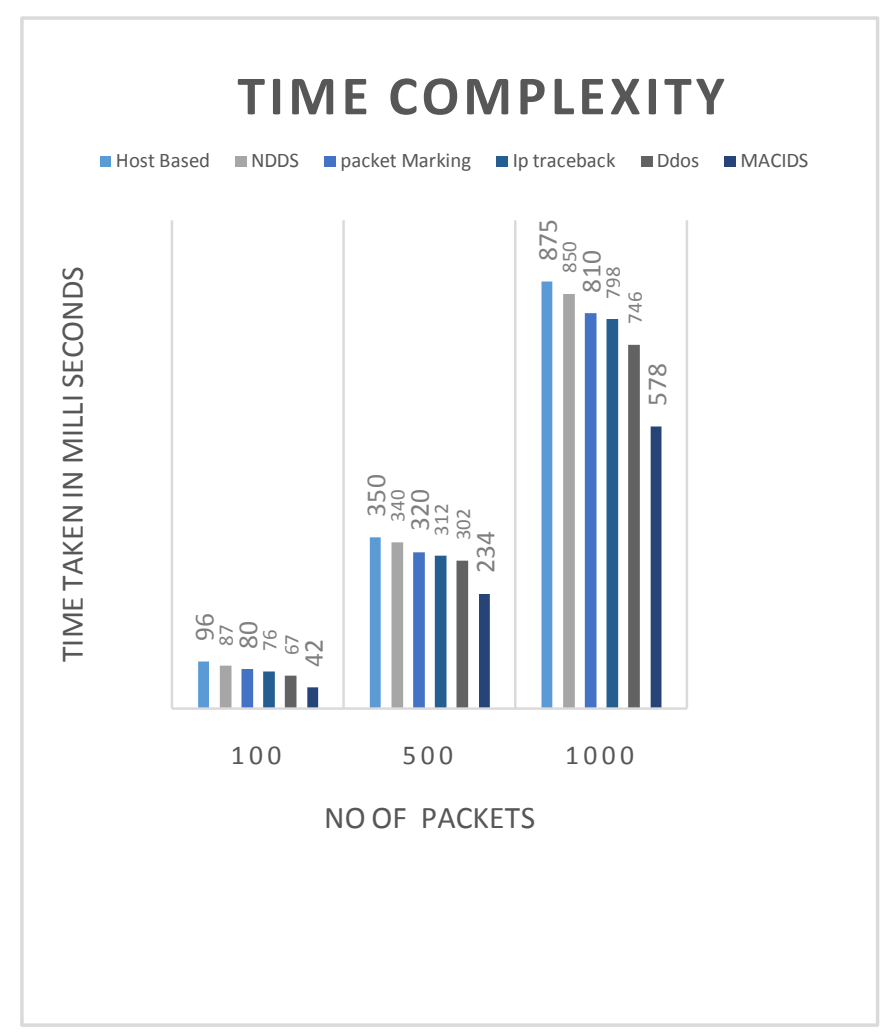

Graph 2: Shows the Time Complexity of the Proposed System 
The graph2 shows the time complexity of the proposed system compare to other methodologies. It shows clearly that the proposed system takes only little time compare to other methods for different number of packets.

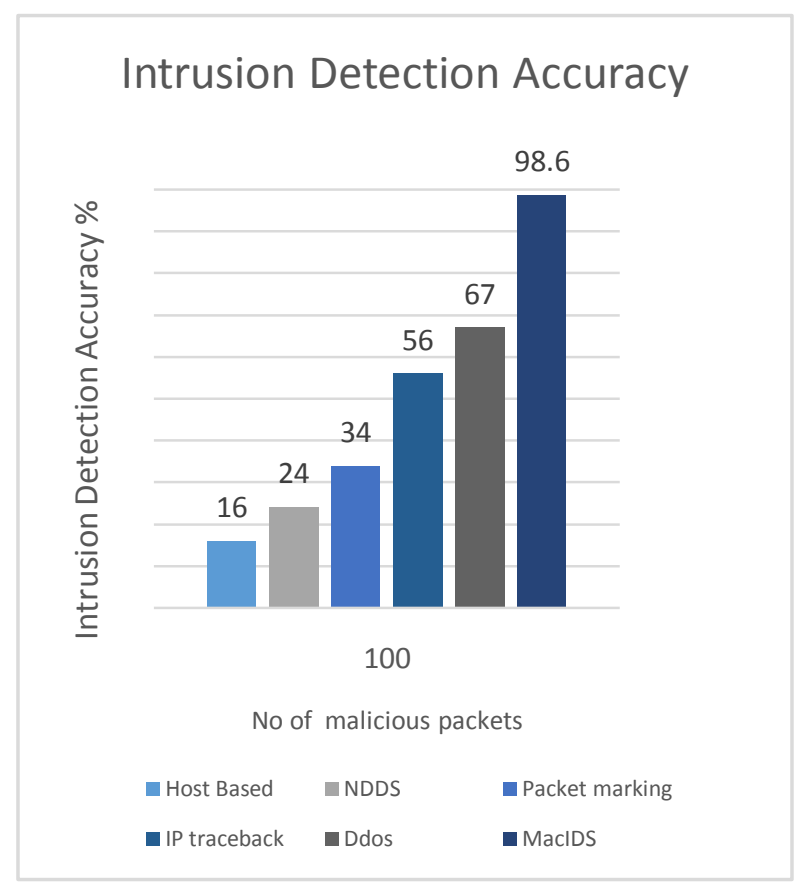

\section{Graph 3: Comparison of Intrusion Detection Accuracy}

The graph 3, shows the comparison of intrusion detection accuracy and it shows clearly that the proposed approach has produced efficient result and produces more accurate results. Intrusion is equipped with a small amount of the snapshot information termed as side information, can infer an extended view of the whereabouts of a victim node appearing in an anonymous trace. Our results quantify the loss of victim nodes' security as a function of the nodal mobility, the inference strategies of adversaries, and any noise that may appear in the trace or the side information. Generally, our results indicate that the security concern is significant in that a relatively small amount of side information is sufficient for the adversary to infer the true identity (either uniquely or with high probability) of a victim in a set of anonymous traces

\section{CONCLUSION}

MANET is susceptible to various intrusion occurrences due to its substructure less or wireless environment to make its communication. To have safe Communication it is must be secure network. There are various attacks in MANET and intrusions differs to create mitigation is one attack which is indistinguishable to identical and smart very unsafe called Sybil attack, it uses frequent independences or uses the individuality of additional node contemporary in the network to disturb the communication or decrease the trust of genuine nodes in the network communication. In this paper we projected to intent the MAC based IDS detection and prevention method which uses MAC Address to detect Sybil nodes to precaution the network communication.

\section{REFERENCES}

[1] A. Nadeem and M.P. Howarth, "A survey of manet intrusion detection \& prevention approaches for network layer attacks”, IEEE Communications surveys and tutorials, Vol. 15, No. 4, Pp. 2027-2045, 2013.

[2] J.R. Douceur, “The sybil attack”, International Workshop on Peer-toPeer Systems, Pp. 251-260, 2002.

[3] C. Piro, C. Shields and B.N. Levine, "Detecting the sybil attack in mobile ad hoc networks", Secure comm and Workshops, Pp. 1-11, 2006.

[4] H. Yu, M. Kaminsky, P. Gibbons and A. Flaxman, "Sybilguard: Protecting against sybil attacks via communal networks", IEEE/ACM Transactions on Networking, Vol. 16, No. 3, Pp. 576-589, 2008.

[5] J. Newsome, E. Shi, D. Song and A. Perrig, "The sybil attack in sensor networks: analysis \& defenses”, Proceedings of the 3rd international symposium on Information processing in sensor networks, Pp. 259-268, 2004.

[6] Roopali Garg and Himikaharma, "Projected Lightweight Sybil Attack Detection Method in MANET", International Journal of Advanced Research in Electrical, Electronics and Instrumentation Engineering, Vol. 3, No. 5, 2014.

[7] N. Joshi and M. Challa, "Secure Authentication Protocol to Identify Sybil Attacks in MANETs", International Journal of Computer Science \& Engineering Technology (IJCSET), Vol. 5, No. 06, 2014.

[8] D. Shehzad, A. Iqbal Umar, N. Ul Amin and W. Ishaq, "A Novel Instrument for Discovery of Sybil Attack in MANETs”, International conference on Computer Science and Information Systems, 2014.

[9] S. Abbas, M. Merabti, D. Llewellyn Jones and K. Kifayat, "Intrusion based Lightweight Sybil Attack Detection in MANETs", IEEE systems journal, Vol. 7, No. 2, 2013.

[10] Y.D. Malkhede and P. Selokar "analysis of sybil attack detection in mobile adhoc network", thirf international conference on proceedings, 2015.

[11] P. Kavitha, C. Keerthana, V. Niroja and V. Vivekanandhan, "Mobile-id Grounded Sybil Attack detection on the Mobile ADHOC Network", International Journal of Communication and Computer Technologies Vol. 02, No. 02, 2014. 